it was found that after the introduction of the recompression chamber the death-rate among the workmen fell from 25 to $1 \frac{1}{2}$ per cent. per annum. To get the best results the men should be placed in the immersion chamber as early as possible after the development of symptoms. In many instances symptoms do not recur after recompression; in other instances relief may or may not be obtained.

The following is an interesting experience of recompression given to me by a patient under my care in the Royal Victoria Infirmary. On the morning of August 6th, 1908, a sinker, aged 25 years, went to work at 6 o'clock and remained under 30 pounds pressure until 9 o'clock. After coming out of the air-lock he felt pains in his back and fainted; he was carried into the medical lock and recompressed in a pressure of 20 pounds. At 10 A.M. he had recovered and preferred to go back to work in the caisson where he remained until 1 P.M., feeling quite well and doing his work as well as the other men. $\mathrm{He}$ had hardly left the air-lock a second time when he was seized with pains in his arms and elbows and was placed in the recompression chamber at a pressure of 30 pounds. After being there for half an hour and feeling relieved the pressure was lowered to 24 pounds, but as the pains returned the pressure was again raised and he remained in the chamber until 6.15 P.M., when he came out free from pain. He left the works and was proceeding homewards when he was seized with pains in the right arm, but he went home, had tea, went out for a short walk, and afterwards went to bed. The pains by this time had become so severe that he conld not sleep. He got up and going back to the works he re-entered the medical lock at 10 P.M., where he stayed until 5 o'clock on the Saturday morning. He slept for four hours in the medical lock and on coming out of the lock was all right, but severe pains developing in his knees he again entered the medical lock at $5.45 \mathrm{~A}$.M. and stayed there until 8.45 A.M. in a pressure of 26 pounds. Shortly after leaving the recompression chamber the pains returned, and as these were severe Dr. Maclay gave him a hypodermic injection of morphine and sent him into the infirmary at Newcastle. The patient, who was a well-developed man, had previously worked under 25 pound pressures in Dublin for six months and in Glasgow for four months under lower pressures without suffering. When admitted into the infirmary he was still in such pain in the knees and elbows that hie had to have another injection of morphine. There could be felt distinct creaking in the skin over the left knee as in emphysema. By degrees the pains lessened and ultimately disappeared. The temperatures for the three evenings after his admission were $100^{\circ} \mathrm{F} ., 100 \cdot 4^{\circ}$, and $99 \cdot 80^{\circ}$. The sounds of the heart which were normal were distant and not at all easily heard.

When recompression cannot be carried out-as, for example, when the symptoms come on long after a man has left the works-he should be put to bed, kept warm, and if in great pain a hypodermic injection of morphine may be administered. In many of these cases, even although there is no paralysis of the limbs, there is frequently a paralytic con dition of the bladder attended by retention of urine and calling for the use of the catheter. Muscular paralysis, loss of sensation, bed-sores, and complications must be treated on ordinary lines.

Since the risks to life from rupture of a caisson are enormous, the greatest care should be taken to prevent such an accident. Whether the shifting of the surface of the river's bank towards the Tyne at Hebburn on account of the excessive dredging of the river so pressed upon the caisson and cracked it, or whether there had occurred a sudden lowering of pressure followed by a jerk of the caisson which broke its attachments, it is difficult to say, but engineers are agreed that caissons should be made of strong steel or of wrought-iron plates and not of cast iron, and that these chambers should be made of such strength as to bear two or three times greater pressure than is ever likely to be required of them.

Royal Society of Arts: Swiney Prize.A meeting of the adjudicators of the Swiney Prize was held at the Royal Society of Arts on Jan. 20th, when, on the motion of the Lord Chief Justice, seconded by Sir R. Douglas Powell, the prize was awarded to Dr. Charles A. Mercier for his work, "Criminal Responsibility."

\section{"BACILLUS F": AN ORGANISM OBTAINED IN A CASE OF EPIDEMIC DIARRHCEA.}

\section{BY J. ORR, L.R.C.P. HDIN.,}

R. STENHOUSE WILLIAMS, M.B., C.M. EDIN., AND

H. LEITH MURRAY, M.D. ABERD.,

OF THE DEPARTMENT OF HYGIENE AND BACTERIOLOGY, UNIVERSTTY OF IIVERPOOL;

$$
\text { AND }
$$

C. RUNDLE, M.D. LOND., AND

A. E. WILLIAMS, M.D. EDIN.,

OF THE LIVERPOOL CITY HOSPITAI, FAZAKERLEY.

IN the summer of 1907 it was decided to admit cases of epidemic diarrhoea into the Liverpool City Hospital at Fazakerley. The number of cases which came under observation was relatively small owing to the wetness of the season, but from one of them, a case presenting all the clinical features of the disease, the organism which is the subject of this paper was obtained. For convenience we have called it " Bacillus F."

A review of the many unsatisfactory attempts to isolate a specific organism from the frees led us to adopt the method of blood culture-the more so as this has proved useful in other diseases. Seven cases were investigated, six of them during life. In these about four cubic centimetres of blood or as much as was available, were received directly from a vein in the forearm into a syringe containing an equal amount of a sterile solution of 1 per cent. citrate of sodium in 0.75 per cent. saline solution. The mixture was immediately inoculated into ascitic fluid broth, ox-bile, milk, blood serum, agar and MacConkey tubes, and incubated at $35^{\circ} \mathrm{C}$. The blood obtained from five of the cases proved sterile in all media. The sixth showed a short motile bacillus in one ascitic fluid broth tube; unfortunately, this organism died out in spite of frequent subculture before we had time to investigate it thoroughly. In the seventh case, immediately after death the heart was seared, a sterile pipette was introduced, and blood was withdrawn and inoculated on to agar slant tubes. After incubation at $35^{\circ} \mathrm{O}$. a pure culture was obtained of the bacillus $F$.

Morphology. - The organism is a short cocco-bacillus, highly motile and flagellated. The flagella are attached near the ends of the bacillus and are shorter and thicker than those of bacillus typhosus. The well-marked flagella and high motility readily differentiate it from the bacilli of Flexner and Shiga.

Staining reactions. - It retains the ordinary simple stains but is decolourised by Gram's method.

Cultivation experiments. - The bacillus $\mathrm{F}$ grows well on all ordinary media at the usual hot and cold incubator temperatures. Anaerobic cultures by the pyrogallic method at $35^{\circ} \mathrm{C}$. were negative. Comparative culture tests were made in various media, the most important of which are tabulated in Chart A. From this chart it is seen that after seven days' incubation at $35^{\circ} \mathrm{C}$. $\left(20^{\circ} \mathrm{C}\right.$. in the case of the gelatin media) the bacillus $F$ differs from the bacillus typhosus, the paratyphoid bacillus $A$ and $B$ (Schottmüller), the bacillus enteritidis Gaertner, and the colon bacillus in the complete absence of any naked-eye change in litmus milk. Even when the tubes were kept in the incubator for three weeks or more no visible alteration took place. We regard this as a most valuable differential test. The absence of indol in peptone water and of fluorescence in neutral red broth is sufficient to separate bacillus $F$ from the colon bacillus. From the bacillus enteritidis Gaertner and the paratyphoid bacilli $A$ and $B$ (Schottmüller) it is distinguished by the absence of fluorescence in neutral red broth; from Morgan's No. 1 bacillus by the absence of indol formation; and from Morgan's bacilli Nos. 3 and 4 by the fact that the bacillus $F$ shows well-marked motility. In order further to differentiate between bacillus $\mathrm{F}$ and allied organisms and to avoid, so far as possible, errors in the reactions due to the introduction of extraneous organic material the following medium was prepared :-
Peptone (Witte)..
Carbohydrate...
1 gramme.
10 per cent. watery solution of litmus ... 10 cubic centimetres.

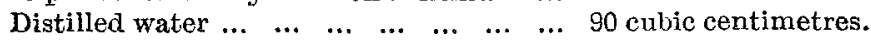


To this medium various carbohydrates were added, and the presence or absence of gas formation was demonstrated by the introduction of Durham's tubes. An uninoculated series was kept and incubated with the inoculated tubes. It was found that the depth of colour in the lactose, mannite, maltose, inulin, and arabinose control tubes became slightly reduced on keeping but not sufficiently to vitiate the experiment.

The tubes were incubated for nine days, but no obvious changes occurred after the fourth day. In Chart $B$ the alterations in the media are epitomised. The reactions obtained at once differentiate the bacillus $\mathbf{F}$ from the bacillus typhosus by the formation of gas in mannite, maltose, dulcite, sorbite, arabinose, and glucose; from bacillus enteritidis Gaertner by the absence of action upon lactose and galactose ; and from the paratyphoid bacilli $A$ and $B$ by the absence of action on galactose. From Morgan's No. 1 bacillus it is differentiated by the formation of gas in mannite, maltose, sorbite, and arabinose, and the absence of gas formation in galactose.
The fact that bacillus $F$ fails to produce any effect upon lactose and galactose is confirmatory evidence of the milk culture reactions previously mentioned.

Agglutination reactions. - A rabbit was given an intraperitoneal inoculation of an emulsion in 0.75 per cent. saline solution from a 24 hours' growth on agar. Diarrhœe was present on the next day and lasted for four days. Blood was obtained from the marginal vein of the ear on varying dates from 21 to 68 days after the inoculation and that of a normal rabbit was used as a control. All the observations were made with 24 hours old lemco broth cultures by means of a hanging drop, one-sixth lens, at laboratory temperature. In the lower dilutions the loop method was adopted. Each dilution formed an individual experiment as it was found that agglutination occurred with great rapidity. To obtain the higher dilutions a graduated pipette was used, so that the serum might be immediately added to the emulsion in the strength required for the experiment. Errors due to the influence of lower dilutions were thus avoided. Two hours were adopted as a time limit. The results of the experiments are seen in

Char'T A.-Comparative Reactions after Seven Days' Incubation.

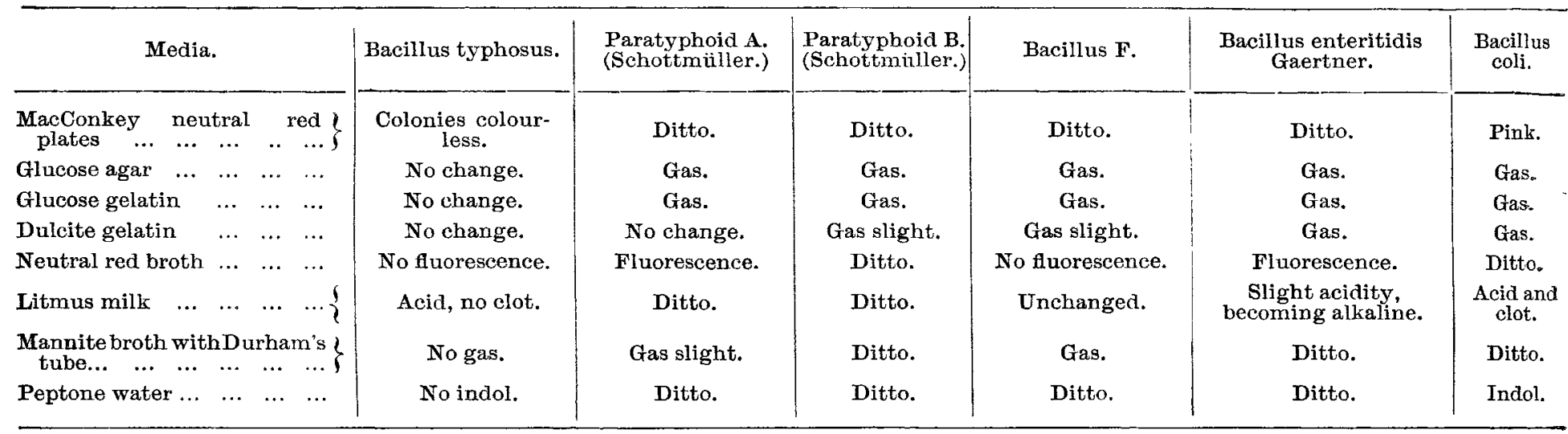

CHART B.-Carbohydrate Reactions.

\begin{tabular}{|c|c|c|c|c|c|c|c|c|c|c|c|c|c|c|c|}
\hline Organism. & 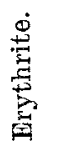 & 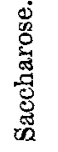 & 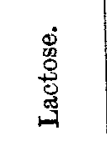 & 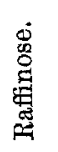 & 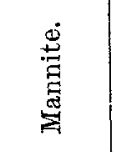 & 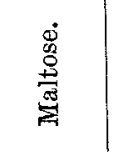 & $\begin{array}{l}\dot{0} \\
\stackrel{0}{0} \\
\stackrel{0}{0} \\
0\end{array}$ & 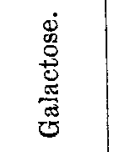 & 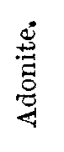 & 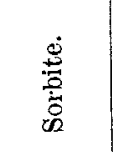 & $\underset{\Xi}{\stackrel{g}{\Xi}}$ & 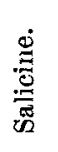 & 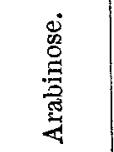 & 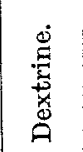 & 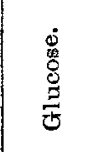 \\
\hline $\begin{array}{llllll}\text { Bacillus F } & \ldots & \ldots & \ldots & \ldots & \ldots\end{array}$ & - & - & - & - & 1. A.G. & $\begin{array}{l}\text { 1. A.g. } \\
\text { 2. A.G. }\end{array}$ & 1.A.G. & - & - & 1.A.G. & 1. A. & - & 1. A.g. & 1. a? & 1. A.G. \\
\hline $\begin{array}{llll}\text { Bacillus typhosus } & \ldots & \ldots & \ldots\end{array}$ & - & - & - & - & 1. A. & 1. A. & - & 1. A. & - & 1. A. & 1. A. & - & - & 1. a. & $1 \mathrm{~A}$. \\
\hline $\begin{array}{cccc}\text { Bacillus paratyphoid } & & \text { A } \\
\text { (Schottmuiller) } & \ldots & \ldots & \ldots\end{array}$ & - & - & - & - & $\begin{array}{l}\text { 1. A.g. } \\
\text { 2. A.G. }\end{array}$ & 1. A.g. & $\begin{array}{l}1- \\
2- \\
\text { 3. A. } \\
\text { 4. A.G. }\end{array}$ & $\begin{array}{l}\text { 1. A. } \\
\text { 2. A.G. }\end{array}$ & - & $\begin{array}{l}\text { 1. A.g. } \\
\text { 2. A.G. }\end{array}$ & $\begin{array}{l}\text { 1. A. } \\
\text { 2. A. } \\
\text { 3. A. } \\
\text { 4. A.g. }\end{array}$ & - & $\begin{array}{l}\text { 1. A. } \\
\text { 2. A.g. } \\
\text { 3. A.g. } \\
\text { 4. A.G. }\end{array}$ & 1. A. & 1. A.G. \\
\hline $\begin{array}{cccc}\text { Bacillus paratyphoid } & \text { B } \\
\text { (Schottmüller) } & \ldots & \ldots & \ldots\end{array}$ & - & - & - & - & 1. A.G. & 1. A.G. & $\begin{array}{ll}\text { 1. a. } \\
\text { 2. } & \text { A.G. }\end{array}$ & 1. A. G. & - & 1. A.G. & 1. A.:G. & - & 1. A.G. & 1. a. & 1 A.G. \\
\hline Bacillus enteritidis Gaertner. & - & - & $\begin{array}{l}\text { 1. a. } \\
\text { 2. - } \\
\text { 3. Alk. }\end{array}$ & - & 1 A.G. & 1. A.G. & $\begin{array}{l}\text { 1. A. } \\
\text { 2. A.G. }\end{array}$ & 1. A.G. & - & 1. A.G. & 1. A.?G. & - & $\begin{array}{l}\text { 1. A.g. } \\
\text { 2. A.g. } \\
\text { 3. A.g. } \\
\text { 4. A.G. }\end{array}$ & - & 1. A.G. \\
\hline Morgan's No. 3 bacillus.* & - & - & - & - & - & - & - & A.g. & - & - & - & - & - & - & A.g. \\
\hline
\end{tabular}

* These reactions are taken from Dr. Morgan's paper in the British Medical Journal, April 21 st, 1906.

The numerals represent the days. $-=$ No change. $\mathrm{A}=$ Acid. a = Slight acid. $\mathrm{G}=\mathrm{Gas}$. $\mathrm{g}=$ Slight gas. Alk. = Alkaline.

ChART C. - Aggtutination Reactions.

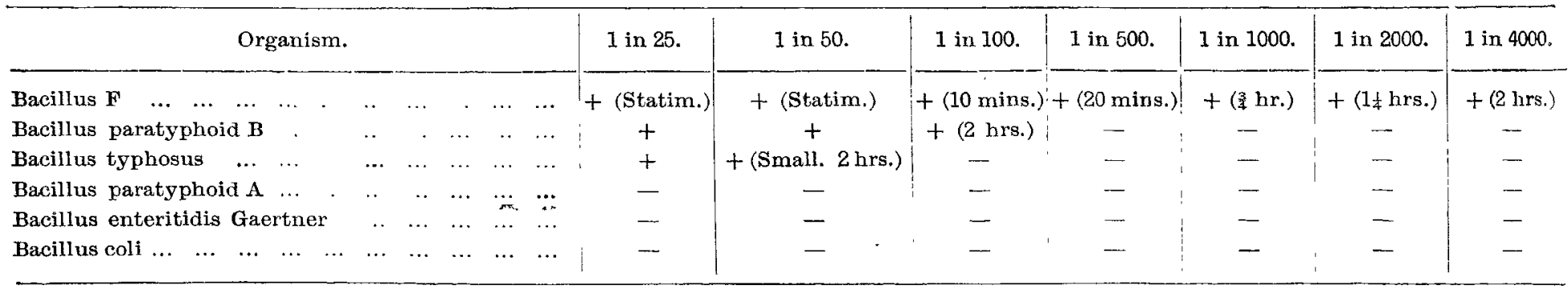


Chart C. From these it appears that the bacillus $F$ is related to the paratyphoid bacillus $B$ and in a much less degree to the bacillus typhosus. With the paratyphoid bacillus $A$, the bacillus enteritidis Gaertner, and the colon bacillus, the agglutinations were negative even in the lowest dilutions. A second rabbit dying in six days showed agglutinative power up to 1 in 100 . Comparative tests were not tried.

Animal Inoculations.

Mice.-Three mice were inoculated intraperitoneally, the first two from the original culture, the third from a culture recovered from mouse No. 2. All died within 24 hours. No. 1 showed green diarrhoea one and a half hours after injection. No such appearance was observed in either of the other two, but post mortem (as will be seen below) the small intestines were filled with fluid green contents. Pure cultures were obtained from the blood in each case after death.

Rats.-Two were inoculated. One died five days later From the fatal case the bacillus was recovered from the blood after death. There was no diarrhœa, but post mortem the intestines were found intensely congested and full of gas and a greenish-yellow fluid. The bronchial glands, however, showed caseous degeneration of old standing; much stress, therefore, cannot be laid on this experiment. The second rat, showing no symptoms after four days, was killed, but nothing was recovered from the blood.

Guinea-prgs.-16 guinea-pigs were inoculated as follows: four from the original strain, one from a culture recovered from a mouse's blood, and 11 from cultures recovered from guinea-pigs. All died within 24 hours and from all pure cultures were again obtained. None showed any diarrhœea, but here again the post-mortem examination showed the fluid green contents of the small intestine observed in the mice. In one case the surface of the mucous membrane of the intestine was washed, seared, and a deep scraping inoculated on to media when a pure culture of the bacillus $F$ was obtained. Two guinea-pigs were inoculated directly with the peritoneal fluid from dead injected guinea-pigs. One died and a pure culture was obtained from the blood and peritoneal fluid; the other was alive and healthy 19 days later.

Post-mortem appearances. - These apply to both mice and guinea-pigs and were found invariable. The parietal peritoneum showed no congestion, but slight effusion was always present. The small intestine, especially in the middle third, was full of a greenish watery fluid, distended with gas and much congested. The spleen and liver were enlarged and congested. The mucous membrane of the small intestine was thick and covered with a glairy effusion. The cæcum was distended with gas; the large intestine usually contained semi- or fully-formed fæces. The pleuræ and lungs appeared normal. The pericardium was normal and there was no excess of pericardial fluid.

Microseopical examination.-Middle third of small intestine: Great congestion of the mucous membrane with a few smail hrmorrhages; the glands showed inflammatory changes, especially towards the lumen of the bowel, and were filled with débris. Lower third of small intestine: Similar changes but less marked. Spleen : Extreme congestion and commencing degeneration of the cells, especially those adjacent to the dilated vessels. A bacillus morphologically resembling bacillus $F$ was seen in the mucous and submucous coats of the middle and lower thirds of the small intestine and in the spleen.

Rabbits. - Four rabbits were inoculated intraperitoneally with varying quantities of the bacillus $F$. Of these one developed no symptoms, one became sick, and two developed diarrhoa. The history of these two is as follows. Rabbit No. 1: Inoculated intraperitoneally on Nov. 26th, 1907. Diarrhœa for four days; rabbit well thereafter. On Dec. 17th and 20th, 1907, and on Feb. 2nd, 1908, comparative agglutination reactions were tested. The result of one of these (Feb. 2nd, 68 days after inoculation) is seen in Chart $\mathrm{C}$. Rabbit No, 2: Inoculated intraperitoneally on July 15th, 1908. Ill July 18th, diarrhœa July 20th, marked emaciation on July 21st, death occurring on the same day. Agglutination at laboratory temperature two hours, $\frac{1}{6}$ lens, 1 in 50 complete in two hours, 1 in 100 marked but not complete in the same time. The bacillus $F$ was recovered from the blood, peritoneal exudate, and in scrapings from a Peyer's patch. The post-mortem appearances were as follows.
Rigor mortis was present, the body was very emaciated, the peritoneum was moderately congested, and the peritoneal luid was in excess; there were some soft fibrinous flakes over the intestines and parietal peritoneum and very marked gaseous distension and considerable congestion of the intestine; the great omentum was shrivelled and fatless, the small intestine contained much greenish fluid, and its walls showed well-marked congestion and prominence of Peyer's patches but no ulceration. The cæcum showed extreme gaseous distension, its contents were semi-fluid and greenish the large intestine was congested in its upper two-thirds and contained no formed masses but gaseous and semi-fluid material. The liver, the spleen, the lungs, the pleuræ, and the pericardium appeared to be normal.

Microsonpical examunation.-Upper third of small intestine: The mucous membrane showed intense inflammatory change and was in parts necrosed and sloughing off; the epithelium where still intact was granular and the nuclei stained feebly or not at all ; the muscular and peritoneal coats were congested. Middle third of small intestine: The mucous membrane was totally disorganised and was replaced by a thin irregular layer of inflammatory round cells and débris ; only occasionally were the tips of crypts seen and these showed intense inflammatory change with commencing necrosis ; the muscular and peritoneal coats were congested. Large intestine: Less destructive change was noticeable here, but the mucous membrane was inflamed and the glands were filled with débris; all the coats were congested. A bacillus morphologically resembling bacillus $F$ was seen in large numbers in the wall of the small intestine and to a much less extent in the large intestine.

Feeding experiments.-Feeding experiments were carried out on a mouse, a rabbit, a kitten, and seven puppies. In the mouse and rabbit there was no result. A three months old kitten was twice fed with the same milk culture as puppy No. 3 without result. Puppy No. 1, aged about six weeks, was fed on April 28th with a very minute quantity of a 24 hours old agar culture emulsified and placed on bread. On April 29th there was slight, and on the 30th marked, diarrhoea, geenish in appearance. This continued until. May 4th, when the puppy had recovered. A control remained well. On May 5th puppy No. 1 was again fed and also a new puppy (No. 2), aged about seven weeks. Each received two tubes of a 24 hours old milk culture. On the 6th puppy No. 1 had violent diarrhoea with marked tenesmus, which continued till the 14th when it began to recover. Puppy No. 2, on the 6th, had very severe diarrhoea, rapidly wasted, and died on the 7 th

On May 8th puppy No. 3 was fed on two tubes of a 24 hours old milk culture. On the 11th it developed diarrhœa, which became very marked on the 12th and lasted till the 23rd. The stools were not well formed until the beginning of June. From the fæces of this puppy the organism was recovered. A loopful was taken into ox-bile and incubated over night, subcultured into lemco broth, and from this plated on to MacConkey bile salt plates. The colourless colonies were picked off, subcultured on agar, and the bacillus was found. The post-mortem appearances found in this puppy are given hereafter.

On July 16th, 1908, puppies Nos. 4 and 5, aged three weeks, were each given two tubes of a 24 hours old milk culture of the original strain (ten months old). On the 26th both showed marked diarrhœa which lasted about a week; there was considerable loss of flesh. No attempt was made to recover the organism from the fæces.

On July 16th, 1908, puppies Nos. 6 and 7, aged three weeks, received the same quantity of a 24 hours old milk culture recovered from a recently dead guinea-pig. Severe diarrhoea appeared on the 21st. On the 28th the diarrhoea was diminishing. On this date one of the puppies was again fed on six tubes oi a 24 hours old milk culture which had been recovered from a guinea-pig on the 20th. On the next dary (29th) this puppy was much worse, diarrhœa was severe and constant, and the puppy could scarcely stand. The other puppy remained as on the 28th. On August 8th the organism was recovered from the fæces of both. Diarrhœa was still present in both on the 11th. The one which received the second feeding was very weak. From that date they gradually improved. Controls from the same litter remained bealthy. On the 25 th the agglutination reaction up to 1 in 25 was positive.

Post-mortem apyearances in puppy No. 2.-The puppy was 
markedly emaciated, the parietal peritoneum was not congested, and there was no effusion into the cavity. The liver and the spleen were normal ; the gall-bladder was full. The intestines as a whole were congested and the small intestine showed dark purple patches. The contents from the duodenum to the rectum were fluid, of a dark green colour in the small intestine and of a yellowish green in the large. There was considerable gaseous distension. The lungs, the pleuræ, and the pericardium appeared normal. Cultures made from the blood were sterile, and those obtained from the intestinal contents and from deep scrapings of the mucous membrane failed to show the specific organism. Sections were made of the intestinal wall and they gave the following results. Duodenum: Some congestion and cedema of the mucous membrane, especially towards the lumen of the bowel. Jejunum: Congestion throughout the depth of the mucous membrane; swelling, granulation, and breaking up of the epithelium towards the lumen of the bowel; débris in the glands ; some small round-celled deposit in the interglandular connective tissue. Ileum : Condition rather more marked than in the jejunum ; a few interstitial small hæmorrhages in the mucous membrane. Large intestine: Congestion of the mucous membrane and a little round-celled deposit in the interglandular connective tissue. A bacillus morphologically resembling the bacillus $F$ was seen in the ileum.

\section{Experiments to Demonstrate the Presence or Absence of Extrasellular and Intracellular Toxins.}

In order to demonstrate the presence or absence of intracellular and extracellular toxins two sets of experiments were devised.

Intracellular toxin.-Inoculations of emulsions of agar cultures in 0.75 per cent. sodium chloride were given after sterilisation at $60^{\circ} \mathrm{C}$. for 40 minutes. The sterility was tested by free inoculation of agar tubes and incubation at $35^{\circ} \mathrm{C}$. The results were as follows. On April 6th a 48 hours old agar culture was emulsified in 10 cubic centimetres of normal saline solution. Of this five cubic centimetres were inoculated intraperitoneally into a guinea-pig. On the next day the animal died. The post-mortem examination disclosed that the middle part of the small intestine was greenish in colour and contained much gas and bile-stained fluid. Films from the peritoneal exudate, the blood, the liver, and the spleen were negative, and cultures from these also proved sterile. On April 9th a 24 hours old agar culture was again emulsified in 10 cubic centimetres of normal saline solution and one cubic centimetre of this was inoculated intraperitoneally into a guinea-pig. On the next day the animal was listless, but it thereafter recovered.

Extracellular toxin. - 700 cubic centimetres of Martin's broth, contained in a Winchester quart bottle laid horizontally in the incubator to give as much air space as possible, were inoculated on the surface and incubated at $37^{\circ} \mathrm{C}$. for 10 days. No surface growth occurred, but an abundant growth formed in the medium. The broth was filtered and tested for sterility. On March 31st five cubic centimetres were inoculated intraperitoneally into a guinea-pig. The result was entirely negative, the guinea-pig remaining without any symptom of illness. So far as these experiments go endotoxins are present and extracellular toxins are absent.

Virulence. - An attempt to gauge any variation in virulence due to passage through a series of guinea-pigs was made by inoculations of 24 hours old agar cultures carefully standardised on the Thoma-Zeiss stage by the method elsewhere described by us. ${ }^{1}$ Eight inoculations were given and in each case where death resulted and in the first case where the animal was killed and the organism grown from the blood, the culture recovered was employed for the next experiment in the series. The results obtained indicate no appreciable rise or fall in the virulence. To insure death a dose of about $2,000,000,000$ was required at the end as at the beginning of the experiments.

Immunity produced. - Incidentally some indication of the production of immunity was given by a guinea-pig in the above series. On June 4 th it received $850,000,000$ of a 24 hours old culture intraperitoneally. On the 5th it was listless but recovered. On the 9 th it was re-inoculated with $2,400,000,000$ of a 24 hours old culture from the same source as the former. The animal remained perfectly well ; control guinea-pigs inoculated with the same dose died.
Conolusions.

The bacillus $\mathbf{F}$ was obtained from a case of epidemic diarrhœa. By its cultural reactions it is readily differentiated from the bacillus typhosus. The absence of indol formation separates it from Morgan's No. 1 bacillus. The presence of well-marked motility is sufficient to distinguish it from Morgan's bacilli Nos. 3 and 4 and from the dysentery bacilli. A reference to the agglutination reactions (Chart $\mathrm{C}$ ) shows that there is a relationship between this organism and the bacillus typhosus and the paratyphoid bacillus $B$. The bacillus $F$ is able to produce diarrhœea in animals and can be recovered from their stools. We believe therefore that it may be an agent in the production of epidemic diarrhoea.

During the summer of 1908 we have further investigated this disease and find that we have obtained a positive agglu. tination reaction varying from 1 in 25 to 1 in 100 in nearly half of the cases tested against the bacillus $F$. The specificity of this reaction we cannot yet affirm, but we hope later to publish a further report on the material obtained during the past summer.

\section{A CASE OF PRIMARY SQUAMOUS-CELLED EPITHELIOMA OF THE EPIDIDYMIS.}

BY R. P. ROWLANDS, M.S. LoND., F.R.O.S. ENG.,

ASSISTANT SURGEON TO GUX'S HOSPITAL AND SURGEON IN CHARGE OF THE ORTHOPEDIC DEPARTMENT; AND

G. W. Nicholson, M.A., M.D. CantaB., GULA SIUDENT OF PATHOLOGY AND DEMONSTRATOR OF MORBID HISTOLOGY, GUY'S HOSPITAL.

As we have been unable to find a similar case recorded we feel that the following is of such unusual interest that we consider ourselves justified in publishing it in full.

A police constable, aged 46 years, a patient of Mr. R. P. Marshall of Bermondsey, was admitted into Guy's Hospital on April 30th, 1907, for a " painful enlargement of the right testicle." During his 25 years' service in the police force he had suffered from influenza nine times, from quinsy once, and from pneumonia once. He said that his right testicle had always been a little larger than the left. There was no history of gonorrhcea or syphilis. On Christmas eve,
1906 , he arrested a drunken man, who grasped and squeezed his right testicle rather severely, but no swelling resulted. A few weeks later he noticed a dragging pain in the testicle which was slightly swollen. The swelling gradually increased in size and became harder. On March 12th, 1907, he consulted Mr. Marshall, complaining of "a feeling of weight, a dragging pain in the right testicle, and of occasional vomiting." These symptoms became worse when the patient was standing. A diagnosis of chronic orchitis was made, but in spite of treatment by strapping, mercurial inunctions, and other local applications the swelling persisted and the man began to waste and to have more severe pain. Mr. Marshall noticed some softening at one spot and then favoured the diagnosis of tuberculous disease, and referred him to one of us at Guy's Hospital on April 18th.

At this time the right half of the scrotum was moderately enlarged and contained a swelling which was smooth, soft, and translucent in front and below but hard and fairly irregular posteriorly, where an enlarged epididymis could be recognised. The testicle itself was not enlarged. The scrotum was slightly adherent to the postero-inferior part of the swelling, which was very tender. The cord was slightly thickened at its lower part and a small, round, hard nodule was felt in it just within the external abdominal ring. At the time the nodule was thought to be in the vas deferens, which was, however, quite normal in size and consistency lower down. The vesiculæ seminales and the prostate were not enlarged or unduly tender. The urine and the act of micturition were normal and there were no signs of pulmonary phthisis. The patient was pale and in poor condition but not thin, his pulse was quick, and he seemed to be more ill than the local condition could well explain. No enlargement of the iliac or inguinal glands could be discovered by careful and repeated examinations. There was no growth upon any part of the skin of the body, in the 